\title{
LXI.
}

\section{Ueber den Kohlenstoff- und Siliciumgehalt des Roheisens.}

\author{
Von \\ Max Buchner, \\ Assistent der Chemic am ständ. St. Joanneum zu Gratz.
}

(A. d. Sitzungsber. der kais. Akademie. XXV.)

Die grossen Schwankungen in den Angaben über den Kohlenstoffgehalt der Eisenhohofenprodukte veranlassten mich, eine Reihe von Kohlenstoff- und Siliciumbestimmungen der verschiedensten Roheisensorten nach einem Verfahren zu unternehmen, welches sich im hiesigen Laboratorium auf Hrn. Prof. Dr. Gottli e b's Veranlassung durch Widtermann als vollkommen verlässig und als derzeit bestes bewährt hat.*) Dieses Verfahren beruht auf der Auflösung des Eisens in Kupferchlorid und Wägung des Kohlenstoffs als Kohlensäure. Man übergiesst zu dem Ende einige Gramme des mässig verkleinerten zu untersuchenden Roheisens mit einer concentrirten wässerigen Lösung von krystallisirtem möglichst säurefreiem Kupferchlorid und überlässt so das Eisen einige Tage hindurch der Einwirkung des Kupferchlorids. In den meisten Fällen ist in der eben angegebenen Zeit das Eisen ohne die mindeste Gasentwickelung unter Zurücklassung einer mit dem Glasstabe zerdrückbaren Masse von Kupfer und Kohlenstoff in Lösung gegangen. Man digerirt es sofort unter Zusatz von Chlorwasserstoffsäure, filtrirt es über ausgeglühtem Asbest und wäscht aus.

Nachdem man es scharf getrocknet, bestimmt man den Kohlenstoffgehalt nach Art der organischen Elementar-

*) Jahrbuch der k. k. geologischen Reichsanstalt, 1853, Nr. 3. S. 498, Jahrb. Liebig und Kopp, 1853, S. 722. 
Analyse durch Verbrennen mit Kupferoxyd unter Anwendung eines Stromes von Sauerstoffgas, wodurch sämmtlicher Kohlenstoff zu Kohlensäure verbrannt und als solche gewogen wird.

Dieses Verfahren unterscheidet sich wesentlich von dem früheren, bei welchem man die Kohlenstoffmenge unmittelbar durch Wägung des Kohlenstoffs bestimmte, was immer zu hohe Resultate liefern musste, da die Kohle stets etwas wasserstoffhaltig ist; andererseits ist es durch Wöhler's*) Entdeckung des krystallinischen Siliciums mehr als wahrscheinlich, dass sich solches auch im Roheisen findet und häufig als Graphit bei der Kohlenstoffbestimmung mit in Rechnung gezogen wurde.

Das obige Verfahren diente sonach zur Bestimmung des Gesammtkohlenstoffgehaltes; die für die grauen und halbirten Roheisensorten so wichtige gesonderte Bestimmung des ungebundenen Kohlenstoffgraphits wurde dadurch bewerkstelligt, dass die gewogene Menge Roheisen mit mässig concentrirter Chlorwasserstoffsäure unter Anwendung von Wärme so lange behandelt wurde, bis keine Gasentwicklung mehr wahrnehmbar war. Die Lösung wurde nun vom ausgeschiedenen Graphit über Asbest filtrirt, dieser mit kochendem Wasser ausgewaschen, dann mit Kalilauge, Alkohol und schliesslich Aether behandelt, welche Agentien eine ziemliche Menge von Kohlenwasserstoffverbindungen aufnahmen, was sich an der Färbung der Flüssigkeiten bemerkbar machte. - Diese beiden letzteren Lösungsmittel wurden bisher meist übergangen und noch in neuester Zeit hat $\mathrm{Abel}^{* *}$ ) eine Reihe von solchen Bestimmungen veröffentlicht, aus dnen zu ersehen ist, dass er sich weder des Alkohols, noch des Aethers bediente. Der auf diese Weise nun möglichst reine Graphit, bei dem sich noch eine entsprechende Menge Silicium

*) Nachrichten d. Gesellsch. Wissensch. zu Göttingen 1856, p. 39 - 44. - Dies. J. 67. 362 .

**) Quart. Journ. of the Chem. Soc. IX, 3. Oct. 1856, p. 202. Dies.

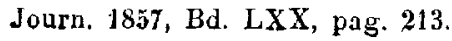


befand, wurde nu: wieder mit Kupferoxyd im Sauerstoffstrome verbrannt und aus der Kohlensäure der Kohlenstoffgehalt berechnet.

Die zur Analyse dienenden Roheisensorten waren nun folgende:

1) Spiegeleisen von Vordernberg von ausgezeichnet krystallinischer Struktur und von grossblättrigem Gefüge.

2) Spiegeleisen, vollkommen krystallinisch.

3) Spiegeleisen von Mosinz von eminent krystallinischem Gefüge.

4) Spiegeleisen von Eberstein, in ganz charakteristischen Stücken.

5) Luckiges Roheisen von Vordernberg, bläulich weiss, von strahlig körnigem Gefüge.

6) Luckiges Roheisen von Plons, Canton St. Gallen, den obigen ganz ähnlich, aus Rotheisenstein mit Holzkohlen erblasen.

7) Weisses Roheisen aus Liezen, Obersteiermark, von strahligem Gefüge.

8) Weisses grelles Roheisen von Liezen, von mehr körniger Struktur.

9) Weisses grelles Roheisen von Liezen, körnig.

10) Weisses grelles Roheisen von Joachimsthal, strahliges Gefüge, durch rasches Erkalten weiss geworden.

11) Halbirtes Roheisen von der Lölling.

12) Stark halbirt erblasenes Roheisen von Liezen.

13) Minder halbirt erblasenes Roheisen von Liezen.

14) Graues Gusseisen von Joachimsthal.

15) Graues Gusseisen von Blansko.

16) Graues Gusseis von Blansko.

17) Schaumiges grobkörniges Roheisen von Blansko.

18) Ueberkohltes, schwarzgrau erblasenes Roheisen von Liezen.

$\begin{array}{llllllllll}\text { Chem. gebund. } & 1 . & 2 & 3 . & 4 . & 5 . & 6 . & 7 . & 8 . & 9 .\end{array}$

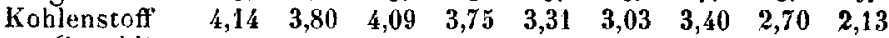

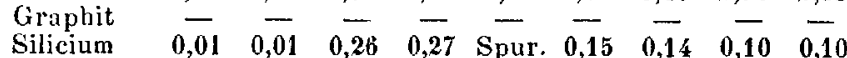

$\begin{array}{llllllllll}\text { Chem. gebund. } 10 . & 11 . & 12 . & 13 . & 14 . & 15 . & 16 . & 17 . & 18 .\end{array}$

$\begin{array}{llllllllll}\text { Kohlenstoff } & \mathbf{3 , 6 0} & \mathbf{3 , 3 4} & \mathbf{2 , 7 2} & \mathbf{2 , 1 7} & \mathbf{1 , 3 5} & \mathbf{1 , 1 8} & \mathbf{0 , 7 1} & \mathbf{0 , 3 8} & \mathbf{0 , 2 6}\end{array}$ $\begin{array}{llllllllll}\text { Graphit } \quad- & - & 0,20 & 2,11 & 2,47 & 2,42 & 2,79 & 3,28 & 3,83\end{array}$ $\begin{array}{llllllllll}\text { Silicium } & 0,66 & 0,10 & 0,26 & 0,09 & 0,7 & 0,66 & 1,53 & 1,62 & 0,59\end{array}$ 
Das Spiegeleisen enthält nach Bromeis*) eine nicht unansehnliche Quantität Graphit, während der Verfasser in verschiedenen Spiegeleisen keinen nachweisen konnte, dagegen fand, dass das gewöhnliche Eisen, namentlich in der Nähe der Lucken oder an den Rändern häufig graues Roheisen eingelagert enthält, es haben aber dann die zunächst liegenden Partien nie das Ansehen des wahren Spiegeleisens, sondern das des dickgrellen weissen Roheisens. Stücke mit grossen Krystall- oder Theilungsflächen enthalten nie Graphit. Es ist daher sehr leicht möglich, dass Bromeis ein solches fälschlich Spiegeleisen genanntes Stück der Analyse unterwarf. Rammelsber g**) hat ebenfalls jene Angaben als einer erneuerten Untersuchung würdig dahingestellt. Schon aus dem bei der Methode der Kohlenstoffbestimmung Berührten geht hervor, dass der Kohlenstoffgehalt des Spiegeleisens im Allgemeinen bisher immer zu hoch angegeben wurde, was auch von der Verbrennung mit Sauerstoffgas herrühren mag, wo in solchen Fällen, wie es scheint, versäumt wurde, den Sauerstoff in den Apparaten durch Luft wieder zu verdrängen, was dann jedenfalls ein fehlerhaftes Resultat liefert. Die übrigen weissen Roheisensorten haben sich ebenfalls als Graphit frei gezeigt, was sowohl die bisherigen Erfahrungen bestätigen, als auch mit derTheorie der Roheisengewinnung übereinstimmt. Der Siliciumgehalt verschwindet beim Spiegeleisen zum Theil beinahe gänzlich, und ist bei dem weissen Roheisen ebenfalls gering.

Die grauen Roheisensorten zeigen einen verbältnissmässig constanten Graphitgehalt. Der aus dem Roheisen erhaltene Graphit wurde früher häufig für eine Verbindung des Kohlenstoffs mit Silicium**) oder auch von Eisen mit Kohlenstoff gehalten. Beides beruht jedenfalls

*) Rammelsberg Lehrb d. chem. Metallurgie, 1850, p. 68.

**) Wehrle, Lehrb. der Probir- und Hüttenkunde, 2. Ausgabe, Bd. 2, p. 19.

**) Rammelsberg Lehrb. d. chem. Metallurgie, 1850, p. 74. 
auf einer irrigen Beobachtung. Wehrle untersuchte demnach wahrscheinlich einen Graphit, dem krystallisirtes Silicium beigemengt war, während im zweiten Falle der Verfasser nach sorgfältiger Digestion des Gemenges von Graphit und Silicium mit Königswasser, nach dem Verbrennen des Graphits vor der Gaslampe mit Gebläse immer vollkommen ungefärbte, eisenfreie Kieselsäure erhielt. Somit fände auch jener fragliche Punkt über die Constitution des Hohofengraphits seine Erledigung, von dem Rammelsberg sagt, dass er eine erneuerte Untersuchung verdiene. Der höhere Siliciumgehalt des grauen Roheisens bestätigt ferner die Ansicht, dass die Reduction des Siliciums erst bei einer Temperatur zu beginnen scheint, wo das Roheisen grau zu werden beginnt. Das Maximum des Siliciumgehalts tritt bei dem schaumigen grobkörnigen Roheisen aus Blansko, wo er 1,6 p. C. erreicht, ein, während Karsten in einem anderen grauen Roheisen als Maximum 3,4 p. C. gefunden hat.

Diesen Untersuchungen zufolge ist man keineswegs berechtigt, eine nur wabrscheinliche Formel für die $\mathrm{Zu}$ sammensetzung des Spiegeleisens aufzustellen und der so einfache Ausdruck des Viertelkohleneisens, welches man bisher als den Hauptbestandtheil des Spiegeleisens betrachtet, scheint demnach kaum gerechtfertigt. Man muss im Gegentheil annehmen, dass das Spiegeleisen die Verbindung eines noch unbekannten Kohleneisens mit reinem Eisen sei, daher es auch auf eine so einfache Formel, wie die des Viertelkohleneisens ergiebt, keinen Anspruch machen kann. Würden nicht die physikalischen Eigenschaften des Spiegeleisens und der Umstand, dass aller Kohlenstoff darin chemisch gebunden enthalten ist, so sehr für die Existenz einer wirklich chemischen Verbindung sprechen, so würde man sich gar nicht veranlasst sehen, darin eine nach stöchiometrischen Verhältnissen bestehende Verbindung anzunehmen.

Indem gerade die ausgezeichnet krystallinische Struktur des Spiegeleisens zur Annahme einer chemischen Verbindung führte, so sind schon von $K$ arsten, $H$ a usmann, Mitscherlich, Rammelsberg und Gurlt 
Krystallmessungen ausgeführt worden, wie auch an einem ziemlich ausgebildeten Krystalle, den ich erhielt, Winkel von ungefähr $86^{\circ}, 105^{\circ}$ und $119^{\circ}$ gefunden wurden, ohne dass man jedoch bezüglich des Krrystallsystemes daraus eine Folgerung machen könnte.

\section{LXII. \\ Neue Titrirmethode des Kupfers.}

Nachdem E. O. Brown die voluminometrischen Methoden Pelouze's und Parkes' zur Ermittelung des Kupfers als zu ungenau befunden hatte, versuchte er eine andere, die befriedigende Resultate lieferte. (Quart. Journ. of the Chem. Soc. April 1857. X, 65.)

Diese Methode besteht in der Zersetzung von Jodkalium durch Kupferoxydsalz und Titrirung des ausgeschiedenen Jods mittelst einer Lösung von unterschwefligsaurem Natron. Der chemische Process dabei ist bekanntlich folgender: $2 \dot{\mathrm{C}} \dddot{\mathrm{S}}$ und $2 \mathrm{KJ}=2 \dot{\mathrm{K}} \dddot{\mathrm{S}}, \epsilon \mathrm{GJ}$ und $\mathrm{J}$; ferner $J$ und $2 \dot{\mathrm{Na}} \ddot{\mathrm{S}}=\mathrm{NaJ}$ und $\dot{\mathrm{NaS}}_{4} \mathrm{O}_{5}$. Das Verfahren des Verf. besteht kurz in nachstehenden Manipulationen:

Man löst die gewogene Menge Kupfer (wenn das Metall ermittelt werden soll) in verdünnter Salpetersäure, kocht bis zum Verschwinden der salpetrigen Säure, verdünnt und setzt kohlensaures Natron zu bis zur eben beginnenden Ausscheidung von Kupferoxyd. Iaann löst man in Essigsäure auf, giebt die hinlängliche Menge Jodkalium zu und wartet bis sich dieses gelöst hat, ehe man mit der titrirten Lösung des unterschwefligsauren Natrons das ausgeschiedene Jod in Lösung bringt: Zur zweckmässigen Ausführung der Titrirung ist es crforderlich, dass zuerst durch einen Antheil der Natronsalzlösung so viel von dem freien Jod gelöst werde, dass die Flüssigkeit nicht mehr braun, sondern nur gelb gefärbt sei, und dass man dann erst die klare Stärkelösung zugebe, welche zur Erkennung

J ourn. f. parkt. Chemie. Ixxif. f. 